\title{
A tolerability and patient acceptability pilot study of a novel antimicrobial urinary catheter for long-term use
}

\begin{tabular}{|r|l|}
\hline Journal: & Neurourology and Urodynamics \\
\hline Manuscript ID & NAU-18-0409.R2 \\
\hline Wiley - Manuscript type: & Original Clinical Article \\
\hline Subject Sections: & Bioengineering \\
\hline Keywords: & $\begin{array}{l}\text { Urinary catheters, catheter-related urinary tract infections, anti-infective } \\
\text { agents, safety, clinical trial }\end{array}$ \\
\hline \multicolumn{2}{|l}{} \\
\hline
\end{tabular}

\section{SCHOLARONE ${ }^{\text {TM }}$ \\ Manuscripts}




\section{Abstract:}

2 Aims:

3 We have developed a novel antimicrobial urinary catheter (AUC) impregnated with

4 rifampicin, triclosan, and sparfloxacin and demonstrated that it has long-term ( 84days)

5 protection against bacterial colonisation in vitro. This study aimed to assess the safety and

6 patient acceptability of this device in long-term catheter users.

\section{Methods:}

9 Adults who use longterm (>28days) indwelling urinary catheters with capacity to consent

10 were invited to receive the AUC at their next catheter change. The primary outcome

11 measure was adverse events $(\mathrm{AE})$ attributable to antimicrobial impregnation of the catheter.

12 Secondary outcome measures included severity of related AEs, patient acceptability, early

13 removal of the trial catheter, and degree of microbial colonisation of trial catheters. Except

14 for the last, outcomes were assessed by telephone interviews. Original and trial catheters

15 were collected, and the lumens and balloons were separated and analysed for

16 microbiological colonisation.

18 Results:

19 Thirty participants were recruited. 84 AEs were reported, and only one was rated as

20 'probably' related to antimicrobial impregnation. The AE was mild and resolved within 48

21 hours. $82.14 \%$ of participants rated the catheter as no different or better than their usual

22 catheter. Two participants chose to remove the AUC early due to it feeling shorter. There

23 were significantly fewer bacterial isolates attached to the balloons of trial catheters

24 compared to the matched original catheters.

Conclusions: 
27 The AUC has an advantageous safety profile and was acceptable to the majority of

28 participants. Information gained from this trial will support a larger randomised controlled

29 study of efficacy.

30

31 Keywords: urinary catheters, catheter-related urinary tract infections, anti-infective agents,

32 safety, clinical trial

33

34

Introduction:

35

Catheter-associated urinary tract infections (CAUTI) are costly for health care

systems as well as distressing for those who suffer from repeated infections and blockages.

37 Long-term indwelling catheter users, who require catheterisation for over 28 days, are

particularly at risk of CAUTI ${ }^{1}$. Two antimicrobial catheters, a silver-alloy coated catheter and

a nitrofurantoin-coated catheter have been commercially available, but a robust randomised

controlled trial has demonstrated that neither significantly reduces CAUTI even in short-term

catheter users. Also the patients who received the nitrofurantoin-coated catheter

experienced greater discomfort than with the control catheter ${ }^{2}$. Therefore, there is no

commercially available anti-CAUTI technology for those who require catheterisation for over 28 days.

We have previously developed a silicone urinary catheters impregnated, not coated, with rifampicin, sparfloxacin, and triclosan and demonstrated seven to 12 weeks of

47 protective activity against colonisation by major uropathogens, including multi-drug resistant 48 strains $^{3}$. The long-term duration of activity is conferred by the migration of the antimicrobials 49 through the silicone to the intraluminal, extraluminal and balloon surfaces. Particularly, in

50 light of the discomfort experienced with the nitrofurantoin-coated catheter, this study aims to 51 understand primarily the tolerability of this novel antimicrobial urinary catheter (AUC) in the 52 target patient population. Specifically, this was determined by the rate of adverse events 53 (AEs) attributable to the antimicrobials or the antimicrobial impregnation process. Other 54 secondary outcomes included patient acceptability, trial withdrawal, severity of AEs, time to 
occurrence of AEs, microbial colonisation of the AUC. The trial was not intended to determine efficacy in reducing CAUTI, but instead to determine in human participants for the first time, the tolerability and acceptability of a novel antimicrobial-impregnated catheter for long-term use.

\section{Materials and Methods:}

\section{Patient and public involvement}

Lay members who were either longterm catheter users or carers were recruited to a research management committee to meet several times yearly to review the trial protocol, trial progress, and trial results. All travel expenses were covered and lay members received payment for attendance.

\section{Manufacture of trial devices}

Two-hundred and five all-silicone, two-way urinary catheters in sizes $12-20 \mathrm{Ch}$ standard and female lengths were impregnated according to a previously published method ${ }^{3-}$ ${ }^{5}$. Briefly, with any plastic ports and connectors removed, and catheters were immersed in a chloroform solution containing $0.2 \% \mathrm{w} / \mathrm{v}$ rifampicin (Sigma-Aldrich), $1.0 \% \mathrm{w} / \mathrm{v}$ triclosan (Irgacare MP, BASF) and 1.0\% sparfloxacin (Sigma-Aldrich) for one hour. The catheters were removed and the chloroform was left to evaporate off under constant air flow for at least 12 hours. Surface residues were removed by rinsing in ethanol and the catheters left to dry. The catheters were packaged in individual plastic sleeves within Tyvek packaging with a clear front and opaque back. The catheters were sterilised by ethylene oxide and removal of ethylene oxide residuals was verified by gas chromatography by a Varian gas chromatograph using a 10 volt detector and $1 \mu \mathrm{L}$ injection volume.

Chloroform removal was verified by gas-chromatography-mass spectrometry in which catheter segments were immersed in acetone to extract the chloroform. Analysis was carried out using a JEOL AccuTOF GCX (Jeol Ltd.) mass spectrometer and an Agilent 
$827890 B$ (Agilent Technologies Inc.) gas chromatograph. An extended ion current trace at 82.9

83 mass to charge ratio $(\mathrm{m} / \mathrm{z})$ was used to detect chlorine isotopes.

84 High-performance liquid chromatography (HPLC) was employed to verify antimicrobial contents of the manufactured trial catheters. Please see Supporting Information Method 1 for the full method of drug content analysis. Briefly, the antimicrobials were extracted in chloroform, resolubilised in methanol, and analysed by an Agilent 1100 HPLC machine with a variable wavelength UV detection (Agilent Technologies Inc).

\section{Participants and setting}

Adults (age 16 years or greater) who were catheterised with a long-term indwelling urinary catheter and who required another long-term indwelling urinary catheter were initially considered for inclusion. Please see Table 1 for the full inclusion and exclusion criteria.

94 Participants were recruited from the community and hospital settings through letters of invitation and screening as participants came through Urology clinics

Study design

This single-centre, non-randomised trial with the aim of evaluating the safety of a CEmarked medical device with modifications was carried out between November, 2016 and February, 2018. Eligible participants who provided informed consent were catheterised with the AUC (trial catheter) at their next scheduled catheter change date. They were catheterised with the AUC for their normal catheterisation length, which ranged from 28-84 days. Participants were interviewed by telephone at 24,48 , and 72 hours postcatheterisation and then once weekly for the rest of the trial duration. The original catheter and trial catheter were collected upon removal for laboratory analysis.

The primary outcome measure was the rate of adverse events (AE) attributable to

107 the antimicrobials or the impregnation process. All AEs were recorded in the case report and 108 a score of severity and a score of relatedness to the AUC was given to each adverse event 109 by the research nurse and adjudicated by the principal investigator. AEs were detected by 
patient self-reporting during the telephone interview and followed up by a review of the patient's notes. Relationship causality was classified as unrelated, unlikely, possible, probable or definite according to the algorithm given by the World Health Organisation ${ }^{6}$.

Further classification grouped AEs as non-serious or serious. An AE was classified as serious (SAE) if it was fatal, life threatening, resulted in hospitalisation or prolonged hospitalisation or resulted in persistent or significant disability or incapacity.

Secondary outcome measures included time to occurrence of adverse events, patient acceptability which was captured by the telephone interviews, whether the trial device was removed before the planned end date of the trial (trial withdrawal), and microorganism colonisation of trial and original catheters.

\section{Laboratory analysis of removed catheters}

Catheters were analysed within 24 hours of removal. The balloon was separated from the lumen using a sterile scalpel and placed into a sterile Universal container and covered with phosphate buffered saline (PBS). The remaining ports on the catheter were also removed with a sterile scalpel and discarded. The remaining luminal tubing was filled with 1-2 mL of PBS, clamped using sterile, straight-jawed surgical clamps, and placed in a sterile container. The balloon and lumen components were sonicated for five minutes at 30 $\mathrm{kHz}$ to detach the bacteria into the surrounding PBS. The luminal tubing ends were cleaned with an alcoholic pre-injection swab and the lumen sonicate was drained into a sterile Bijou bottle (Sterilin). The balloon and lumen sonicates were plated onto cysteine-lactose electrolyte-deficient medium (Oxoid), and incubated overnight at $37^{\circ} \mathrm{C}$. If culture - positive, the colonies were quantified and general microbiological identification performed. If culture negative the plates were incubated for a further 24 hours.

\section{Statistical analysis} Data were analysed and graphs were prepared using GraphPad Prism 7.01 (GraphPad Software Inc., LaJolla California, USA). 
138 Results:

139 Manufacture of trial devices

140 Trial catheters were validated as being free of chloroform, and ethylene oxide

141 sterilisation residuals were within the acceptable range. HPLC verified that the trial catheters

142 were impregnated with $0.080 \% \mathrm{w} / \mathrm{w}( \pm 0.013 \% \mathrm{w} / \mathrm{w}(\mathrm{IQR}))$ rifampicin, $1.084 \% \mathrm{w} / \mathrm{w}( \pm 0.138 \%$

$143 \mathrm{w} / \mathrm{w})$ triclosan, and $0.704 \% \mathrm{w} / \mathrm{w}(0.155 \% \mathrm{w} / \mathrm{w})$ sparfloxacin.

144

145

Participant Demographics

146 Thirty participants were recruited and were catheterised with the AUC. Please see

147 Figure 1 for a STROBE flow diagram of recruitment and participation. The majority of

148 participants were male and except for one patient were catheterised urethrally (Table 2). The

149 mean duration of catheterisation with the trial catheter was 56.03 days with a range of 1-84

150 days. There were a total of 1681 days of participants catheterised with the AUC.

151

152 Primary outcome: safety

153 Eighty-four adverse events were reported by participants (0-11 AEs per participant).

154 The majority (72.62\%) of AEs were 'unrelated' or 'unlikely' to be related to the antimicrobial

155 impregnation process. The AEs considered to be 'possibly' related to the AUC $(26.19 \%)$

156 included blockage of the catheter, CAUTI episodes, and stinging after catheterisation as

157 these are AEs associated with all urinary catheterisation (Table 3 ). The exception to this was

158 one participant who experienced increased stinging following catheterisation that the

159 participant had not experienced with previous catheters. This AE was classified as 'probably'

160 related to antimicrobial impregnation of the catheters due to the noticeable difference

161 between the AUC and the normal catheters. The stinging subsided within 48 hours and the

162 participant then went on to consider the AUC no different from their normal catheter.

163 Patient medical history was recorded at baseline and eight participants had a history

164 of frequent CAUTIs. Four participants each experienced one CAUTI while using the AUC. 
165 This provided a preliminary indication as to the potential efficacy of the AUC, but will need

166 further systematic investigation.

167

Secondary outcome: severity of AEs

None of the AEs with any relationship to antimicrobial impregnation of the catheters was considered serious or severe as seen in Table 3. All SAEs were determined to be

171 'unrelated' to antimicrobial impregnation of the urinary catheters. The participants were not

172 withdrawn from the trial as a result of the SAEs and there were no restrictions on treatments 173 received.

Secondary outcome: time to occurrence of AEs

176 There was no difference between time to $A E$ in the $A E$ causality relationship groups

$177 \quad(p=0.5252$. Log rank test).

178

\section{Secondary outcome: patient acceptability}

$82.14 \%$ of participants rated the AUC as no different or better than their previous catheters and $89.3 \%$ of participants reported the same amount of pain or less pain from the AUC at the last recorded interview (Table 4). becoming infected.

\section{Secondary outcome: trial withdrawal}

192 withdrawn because of catheter expulsion, a burst balloon, or balloon deflation. Bladder 
193 stones were confirmed for the patient who had a burst balloon. One catheter was changed

194 by a district nurse due to concerns that it may have been pulled out of position. The

195 remaining two participants voluntarily ended the trial early, both due to what they felt was a 196 shorter catheter length. However, both had received standard length catheters, which were

$197400 \mathrm{~mm}$ in length. No catheters were removed over safety concerns.

198

199

Secondary outcome: colonisation of original and trial catheters

200 Twenty-nine of the 30 original and 28 of the 30 AUCs were collected. The three lost

201 catheters fell out in the community and could not be retrieved. The original catheters and

202 AUCs were matched ( $n=27$ pairs $)$ and there were significantly fewer $(p=0.0088$, two-tailed $t-$

203 test) species of microorganisms attached to the AUC balloons compared to the matched

204 control catheter. The pairs were well matched for the duration of catheterisation $(p=0.8428$,

205 two-tailed t-test).

206 The lumens of two trial catheters were culture - negative and no lumens of original

207 catheters were culture - negative. One possible concern at the start of the study was that

208 eradication of organisms sensitive to the activity of the AUC would allow replacement by

209 other organisms. However, this was not seen in the catheter analyses. For example, E.

210 faecalis is not sensitive to the activity of the AUC yet there was no overgrowth of $E$. faecalis

211 in the AUCs. In fact, five fewer AUC balloons and two fewer lumens contained E. faecalis

212 compared to the control catheters. In general, the presence of all groups of organisms was

213 reduced in the AUCs, with the exception of Pseudmonas spp. in which there were two more

214 AUC lumens colonised with Pseudomonas spp. compared to the control catheters. The main

215 limitation of this analysis is that the numbers are small and future studies will be needed to

216 monitor the colonising microorganism populations. It is important to emphasise that, though

217 reduction in CAUTI was mentioned by three participants in the free comments (Supporting

218 Information Table 1), this trial was not designed to quantify efficacy.

219

220 Discussion: 
221 Antimicrobial urinary catheters were produced and validated for use in this study,

222 which is the first human trial of this device. 84 AEs in 30 participants across 1681

223 catheterisation days were recorded, and of those only one was identified as being 'probably'

224 related to antimicrobial impregnation. This event was worse stinging than usual at

225 catheterisation and it resolved within 48 hours. The safety profile of the AUC appears

226 favourable. Patient acceptability was positive with $82 \%$ of participants rating the AUC as 'no

227 different' or better than their previous urinary catheters. Of the remaining $18 \%$ (5

228 participants) responding "a bit worse", two felt that their trial catheter was too short, despite it

229 being of identical length to their standard catheter; one changed the type of drainage device

230 and experienced disconnections; one experienced increased urinary urgency, and one

231 experienced pain on passing urine using a catheter valve.

232 Microbiological analysis of the participant's original and trial catheters demonstrated

233 a significant reduction of the number of species attached to the balloon of the AUCs.

234 Importantly, the use of the AUC did not increase the prevalence of MDR organisms or

235 increase the prevalence of microorganisms that are non-susceptible to the activity of the

236 AUC.

237 The CATHETER trial was a multi-centre randomised controlled trial of anti-septic

238 (silver-alloy and nitrofurazone coated) catheters for short-term use. Patients receiving

239 experimental catheters reported increased discomfort following catheterisation (silver

240 catheter $28.7 \%$, nitrofural catheter $38.9 \%$ ) and this was a motivation for our obtaining data

241 on safety and patient acceptability before undertaking larger studies. Our findings regarding

242 comfort and acceptability compared very favourably with these. Haematuria and septicaemia

243 were two recorded significant clinical events included in the CATHETER trial ${ }^{7}$. Haematuria

244 and blockage of the catheter due to a blood clot were recorded during this trial, but there

245 were alternative reasons for the presence of blood such as taking aspirin and the presence

246 of an enlarged prostate which is a recognised cause of haematuria ${ }^{8}$. During adjudication of

247 AE causality, as haematuria was not present without other predisposing factors and was no 
248 worse than previous episodes, the episodes were determined as 'possibly' related as it is 249 related to catheterisation but not necessarily catheterisation with the AUC.

250 The ESCALE trial, a trial of silver-alloy catheters in spinal cord injury patients, 251 reported more AEs possibly related to catheterisation with the experimental catheters 252 compared to standard catheters, including itching which was not reported in the control 253 group ${ }^{9}$. Other AEs captured by the ESCALE trial included haematuria, rash, blockage, and 254 suprapubic pain ${ }^{9}$. Rash related to catheterisation and suprapubic pain were not reported by 255 patients in our AUC safety trial. Blockage was reported during this trial.

256 Other unique AEs reported here included the sensation of needing to void, burning at 257 the beginning and end of passing urine using a catheter valve, difficulty connecting the 258 catheter bag to the catheter, and the catheter drainage system 'pushing off' the catheter 259 connection. While these were mild events and mostly associated with the catheter drainage 260 systems, they were still reported by participants as part of their catheter management. The 261 base silicone urinary catheters that were impregnated with antimicrobials may have been 262 from a different manufacturer than their normal catheter, which could have affected what the 263 catheter user perceives as 'normal' for their catheter.

264 Although the follow-up was short, any AEs relating to the composition of the catheter 265 material are likely to have manifested in the time period studied. Further studies will confirm 266 long-term tolerability as well as clinical efficacy and will benefit from a control arm for 267 comparison. Other limitations include that this was an unblinded study and this may have 268 introduced an element of bias of the participant's in reporting. Although multi-centre trials 269 provide a better generalisability of the results and therefore increased external validity, the 270 participants represented many health conditions and were managed throughout several 271 districts once catheterised with the trial catheter. Therefore, they were managed as standard 272 according to their local policies and guidelines, which were not influenced by the clinical trial.

273 In this trial participants were excluded if they not did have sensation in the urethra 274 and/or bladder as they would be unable to self-report some symptoms and also for their 275 safety. If the AUC were to cause irritation, allergy or discomfort both the participant and 
276 research team would be unaware. This by extension excluded patients with spinal cord injury

277 or cauda equina syndrome. Likewise, many patients with impaired cognitive capacity may

278 require a urinary catheter but were not eligible to participate due to the possible inability to

279 self-report new symptoms or adhere to the telephone interview schedule. These exclusion

280 criteria were put in place to protect patients and to preserve the accuracy of the data

281 collected. They will be included in a further randomised controlled trial of efficacy.

282

283 Conclusions:

284 The AUC has an advantageous safety profile and was an acceptable alternative 285 catheter to the majority of trial participants. Information gained from this trial will support 286 future regulatory applications for commercialisation and larger randomised controlled studies 287 of efficacy of the AUC.

\section{References:}

291

292 1. Loveday H, Wilson J, Pratt R, et al. epic3: National evidence-based guidelines for preventing healthcare-associated infections in NHS hospitals in England. $J$ Hosp Infect. 2014:S1-S70.

2. Pickard R, Lam T, MacLennan G, et al. Antimicrobial catheters for reduction of symptomatic urinary tract infection in adults requiring short-term catheterisation in hospital: a multicentre randomised controlled trial. Lancet. 2012;380(9857):19271935.

3. Fisher L, Hook A, Ashraf W, et al. Biomaterial modification of urinary catheters with antimicrobials to give long-term broadspectrum antibiofilm activity. J Control Release. 2015. 
302 4. Bayston R, Fisher L, Weber K. An antimicrobial modified silicone peritoneal catheter 303 with activity against both Gram-positive and Gram-negative bacteria. Biomaterials. $304 \quad 2009 ; 30(18): 3167-3173$.

305 5. Bayston R, Inventor; University of Nottingham, assignee. Medical devices and $306 \quad$ methods of making medical devices. 2007.

307 6. World Health Organization. The use of the WHO-UMC system for standardizes case $308 \quad$ causality assessment 2002;

309 http://www.who.int/medicines/areas/quality safety/safety efficacy/WHOcausality ass $310 \quad$ essment.pdf. Accessed February, 2018.

311 7. Pickard R, Lam T, Maclennan G, et al. Types of urethral catheter for reducing 312 symptomatic urinary tract infections in hospitalised adults requiring short-term 313 catheterisation: multicentre randomised controlled trial and economic evaluation of 314 antimicrobial- and antiseptic-impregnated urethral catheters (the CATHETER trial). $315 \quad$ Health Technol Assess. Nov 2012;16(47):1-197.

316 8. Vasdev N, Kumar A, Veeratterapillay R, Thorpe AC. Hematuria secondary to benign 317 prostatic hyperplasia: retrospective analysis of 166 men identified in a single one 318 stop hematuria clinic. Curr Urol. 2013;6(3):146-149.

319 9. Bonfill X, Rigau D, Esteban-Fuertes M, et al. Efficacy and safety of urinary catheters 320 321 with silver alloy coating in patients with spinal cord injury: a multicentric pragmatic randomized controlled trial. The ESCALE trial. Spine J. Nov 2017;17(11):1650-1657.

323 Table Legends:

324 Table 1: Inclusion and exclusion criteria

325 Table 2: Participant demographics of consented participants 326 
327 Table 3: Description of adverse events (AEs), their relationship to antimicrobial impregnation

328 of trial catheters and their severity

329

330 Table 4: Replies from last recorded telephone interview of participants

331

332 Figure Legends:

333 Figure 1: STROBE flow diagram of participants involved throughout the trial pathway 334

335 


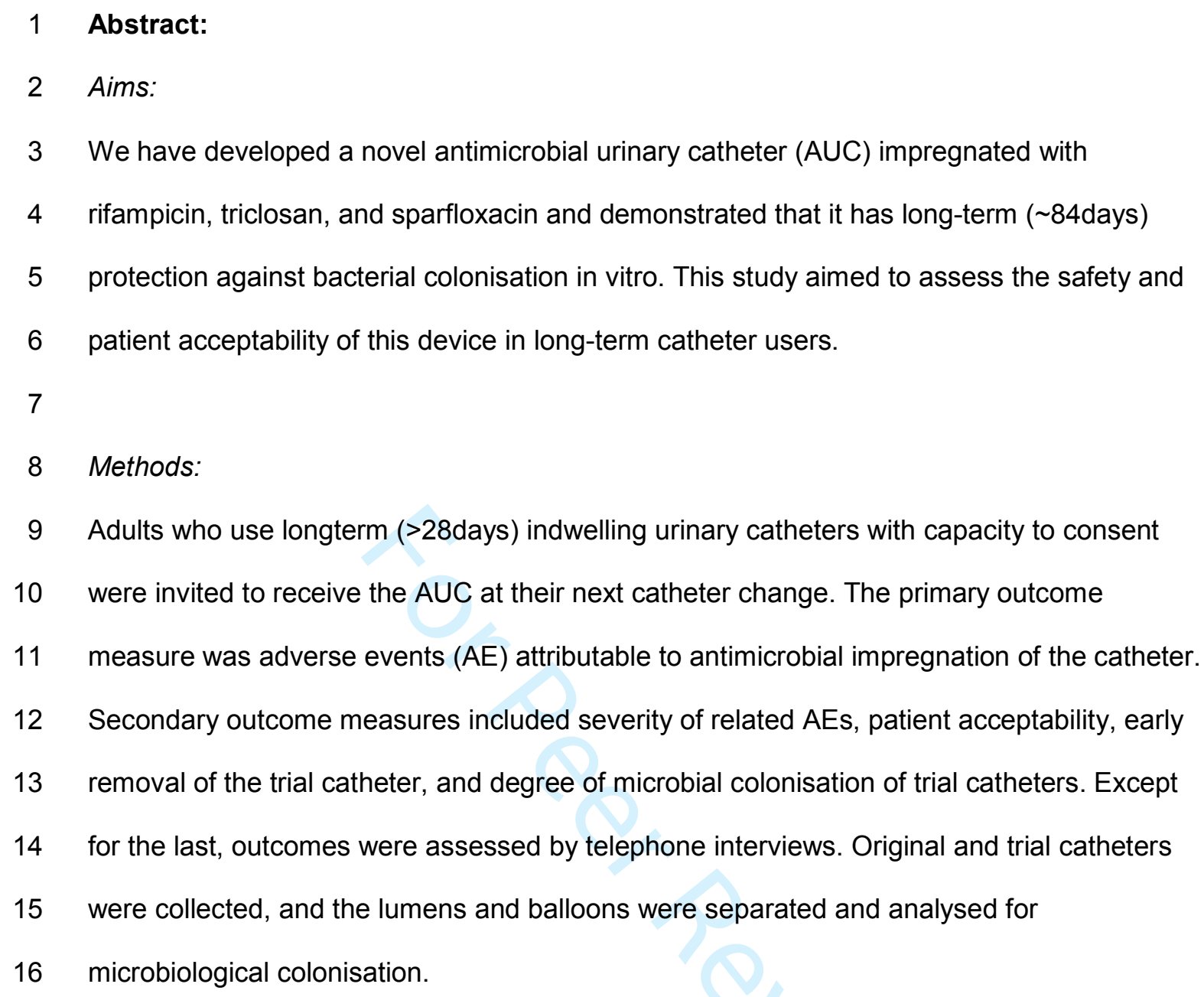

9 Adults who use longterm (>28days) indwelling urinary catheters with capacity to consent

10 were invited to receive the AUC at their next catheter change. The primary outcome

11 measure was adverse events $(A E)$ attributable to antimicrobial impregnation of the catheter.

12 Secondary outcome measures included severity of related AEs, patient acceptability, early

13 removal of the trial catheter, and degree of microbial colonisation of trial catheters. Except

14 for the last, outcomes were assessed by telephone interviews. Original and trial catheters

15 were collected, and the lumens and balloons were separated and analysed for

16 microbiological colonisation.

18 Results:

19 Thirty participants were recruited. 84 AEs were reported, and only one was rated as

20 'probably' related to antimicrobial impregnation. The AE was mild and resolved within 48

21 hours. $82.14 \%$ of participants rated the catheter as no different or better than their usual

22 catheter. Two participants chose to remove the AUC early due to it feeling shorter. There

23 were significantly fewer bacterial isolates attached to the balloons of trial catheters

24 compared to the matched original catheters.

Conclusions: 
27 The AUC has an advantageous safety profile and was acceptable to the majority of

28 participants. Information gained from this trial will support a larger randomised controlled

29 study of efficacy.

30

31 Keywords: urinary catheters, catheter-related urinary tract infections, anti-infective agents,

32 safety, clinical trial

33

34

\section{Introduction:}

Catheter-associated urinary tract infections (CAUTI) are costly for health care systems as well as distressing for those who suffer from repeated infections and blockages. Long-term indwelling catheter users, who require catheterisation for over 28 days, are particularly at risk of CAUTI ${ }^{1}$. Two antimicrobial catheters, a silver-alloy coated catheter and a nitrofurantoin-coated catheter have been commercially available, but a robust randomised controlled trial has demonstrated that neither significantly reduces CAUTI even in short-term catheter users. Also the patients who received the nitrofurantoin-coated catheter experienced greater discomfort than with the control catheter ${ }^{2}$. Therefore, there is no commercially available anti-CAUTI technology for those who require catheterisation for over 28 days.

We have previously developed a silicone urinary catheters impregnated, not coated, with rifampicin, sparfloxacin, and triclosan and demonstrated seven to 12 weeks of protective activity against colonisation by major uropathogens, including multi-drug resistant strains $^{3}$. The long-term duration of activity is conferred by the migration of the antimicrobials through the silicone to the intraluminal, extraluminal and balloon surfaces. Particularly, in light of the discomfort experienced with the nitrofurantoin-coated catheter, this study aims to understand primarily the tolerability of this novel antimicrobial urinary catheter (AUC) in the target patient population. Specifically, this was determined by the rate of adverse events (AEs) attributable to the antimicrobials or the antimicrobial impregnation process. Other secondary outcomes included patient acceptability, trial withdrawal, severity of AEs, time to 
occurrence of AEs, microbial colonisation of the AUC. The trial was not intended to determine efficacy in reducing CAUTI, but instead to determine in human participants for the first time, the tolerability and acceptability of a novel antimicrobial-impregnated catheter for long-term use.

\section{Materials and Methods:}

\section{Patient and public involvement}

Lay members who were either longterm catheter users or carers were recruited to a research management committee to meet several times yearly to review the trial protocol, trial progress, and trial results. All travel expenses were covered and lay members received payment for attendance.

\section{Manufacture of trial devices}

Two-hundred and five all-silicone, two-way urinary catheters in sizes 12-20Ch standard and female lengths were impregnated according to a previously published method ${ }^{3-}$ ${ }^{5}$. Briefly, with any plastic ports and connectors removed, and catheters were immersed in a chloroform solution containing $0.2 \% \mathrm{w} / \mathrm{v}$ rifampicin (Sigma-Aldrich), $1.0 \% \mathrm{w} / \mathrm{v}$ triclosan (Irgacare MP, BASF) and 1.0\% sparfloxacin (Sigma-Aldrich) for one hour. The catheters were removed and the chloroform was left to evaporate off under constant air flow for at least 12 hours. Surface residues were removed by rinsing in ethanol and the catheters left to dry. The catheters were packaged in individual plastic sleeves within Tyvek packaging with a clear front and opaque back. The catheters were sterilised by ethylene oxide and removal of ethylene oxide residuals was verified by gas chromatography by a Varian gas chromatograph using a 10 volt detector and $1 \mu \mathrm{L}$ injection volume.

Chloroform removal was verified by gas-chromatography-mass spectrometry in which catheter segments were immersed in acetone to extract the chloroform. Analysis was carried out using a JEOL AccuTOF GCX (Jeol Ltd.) mass spectrometer and an Agilent 
$827890 \mathrm{~B}$ (Agilent Technologies Inc.) gas chromatograph. An extended ion current trace at 82.9

83 mass to charge ratio $(\mathrm{m} / \mathrm{z})$ was used to detect chlorine isotopes.

84 High-performance liquid chromatography (HPLC) was employed to verify antimicrobial contents of the manufactured trial catheters. Please see Supporting Information Method 1 for

86 the full method of drug content analysis. Briefly, the antimicrobials were extracted in

87 chloroform, resolubilised in methanol, and analysed by an Agilent 1100 HPLC machine with

88 a variable wavelength UV detection (Agilent Technologies Inc).

Participants and setting

Adults (age 16 years or greater) who were catheterised with a long-term indwelling urinary catheter and who required another long-term indwelling urinary catheter were initially considered for inclusion. Please see Table 1 for the full inclusion and exclusion criteria.

94 Participants were recruited from the community and hospital settings through letters of invitation and screening as participants came through Urology clinics

Study design

This single-centre, non-randomised trial with the aim of evaluating the safety of a CEmarked medical device with modifications was carried out between November, 2016 and February, 2018. Eligible participants who provided informed consent were catheterised with the AUC (trial catheter) at their next scheduled catheter change date. They were catheterised with the AUC for their normal catheterisation length, which ranged from 28-84 days. Participants were interviewed by telephone at 24,48 , and 72 hours postcatheterisation and then once weekly for the rest of the trial duration. The original catheter and trial catheter were collected upon removal for laboratory analysis.

The primary outcome measure was the rate of adverse events (AE) attributable to the antimicrobials or the impregnation process. All AEs were recorded in the case report and a score of severity and a score of relatedness to the AUC was given to each adverse event by the research nurse and adjudicated by the principal investigator. AEs were detected by 
110 patient self-reporting during the telephone interview and followed up by a review of the

111 patient's notes. Relationship causality was classified as unrelated, unlikely, possible,

112 probable or definite according to the algorithm given by the World Health Organisation ${ }^{6}$.

113 Further classification grouped AEs as non-serious or serious. An AE was classified as

114 serious (SAE) if it was fatal, life threatening, resulted in hospitalisation or prolonged

115 hospitalisation or resulted in persistent or significant disability or incapacity.

116 Secondary outcome measures included time to occurrence of adverse events,

117 patient acceptability which was captured by the telephone interviews, whether the trial

118 device was removed before the planned end date of the trial (trial withdrawal), and

119 microorganism colonisation of trial and original catheters.

Laboratory analysis of removed catheters

122 Catheters were analysed within 24 hours of removal. The balloon was separated

123 from the lumen using a sterile scalpel and placed into a sterile Universal container and

124 covered with phosphate buffered saline (PBS). The remaining ports on the catheter were

125 also removed with a sterile scalpel and discarded. The remaining luminal tubing was filled

126 with 1-2 mL of PBS, clamped using sterile, straight-jawed surgical clamps, and placed in a

127 sterile container. The balloon and lumen components were sonicated for five minutes at 30

$128 \mathrm{kHz}$ to detach the bacteria into the surrounding PBS. The luminal tubing ends were cleaned

129 with an alcoholic pre-injection swab and the lumen sonicate was drained into a sterile Bijou

130 bottle (Sterilin). The balloon and lumen sonicates were plated onto cysteine-lactose

131 electrolyte-deficient medium (Oxoid), and incubated overnight at $37^{\circ} \mathrm{C}$. If culture - positive,

132 the colonies were quantified and general microbiological identification performed. If culture -

133 negative the plates were incubated for a further 24 hours.

135 Statistical analysis

136 Data were analysed and graphs were prepared using GraphPad Prism 7.01

137 (GraphPad Software Inc., LaJolla California, USA). 
138 Results:

\section{Manufacture of trial devices}

Trial catheters were validated as being free of chloroform, and ethylene oxide sterilisation residuals were within the acceptable range. HPLC verified that the trial catheters were impregnated with $0.080 \% \mathrm{w} / \mathrm{w}( \pm 0.013 \% \mathrm{w} / \mathrm{w}(\mathrm{IQR}))$ rifampicin, $1.084 \% \mathrm{w} / \mathrm{w}( \pm 0.138 \%$ w/w) triclosan, and $0.704 \% \mathrm{w} / \mathrm{w}(0.155 \% \mathrm{w} / \mathrm{w})$ sparfloxacin.

\section{Participant Demographics}

Thirty participants were recruited and were catheterised with the AUC. Please see

Figure 1 for a STROBE flow diagram of recruitment and participation. The majority of participants were male and except for one patient were catheterised urethrally (Table 2). The mean duration of catheterisation with the trial catheter was 56.03 days with a range of 1-84 days. There were a total of 1681 days of participants catheterised with the AUC.

\section{Primary outcome: safety}

Eighty-four adverse events were reported by participants (0-11 AEs per participant). The majority (72.62\%) of AEs were 'unrelated' or 'unlikely' to be related to the antimicrobial impregnation process. The AEs considered to be 'possibly' related to the AUC (26.19\%) included blockage of the catheter, CAUTI episodes, and stinging after catheterisation as these are AEs associated with all urinary catheterisation (Table 3). The exception to this was one participant who experienced increased stinging following catheterisation that the participant had not experienced with previous catheters. This AE was classified as 'probably' related to antimicrobial impregnation of the catheters due to the noticeable difference between the AUC and the normal catheters. The stinging subsided within 48 hours and the participant then went on to consider the AUC no different from their normal catheter.

Patient medical history was recorded at baseline and eight participants had a history of frequent CAUTIs. Four participants each experienced one CAUTI while 
165 using the AUC. This provided a preliminary indication as to the potential efficacy of

166 the AUC, but will need further systematic investigation.

167

168

Secondary outcome: severity of AEs

None of the AEs with any relationship to antimicrobial impregnation of the catheters was considered serious or severe as seen in Table 3. All SAEs were determined to be

171 'unrelated' to antimicrobial impregnation of the urinary catheters. The participants were not

172 withdrawn from the trial as a result of the SAEs and there were no restrictions on treatments 173 received.

Secondary outcome: time to occurrence of AEs

176 There was no difference between time to $A E$ in the $A E$ causality relationship groups

$177 \quad(p=0.5252$. Log rank test).

178

Secondary outcome: patient acceptability

$82.14 \%$ of participants rated the AUC as no different or better than their previous catheters and $89.3 \%$ of participants reported the same amount of pain or less pain from the AUC at the last recorded interview (Table 4).

A full thematic analysis of the free responses can be found as Supporting Information

184 Table 1. At the last interview, three patients reported reduced infections, six commented on

185 increased comfort, three wanted to keep the AUC for longer, and two wanted to have a

186 second AUC. Impacts on mental health included getting better quality of sleep due to less

187 need to empty the bladder via a valve and increased confidence in their catheter not

188 becoming infected.

189

Secondary outcome: trial withdrawal

Nine of the 30 participants ended the trial earlier than expected. Seven were

192 withdrawn because of catheter expulsion, a burst balloon, or balloon deflation. Bladder 
193

194

195

196

stones were confirmed for the patient who had a burst balloon. One catheter was changed by a district nurse due to concerns that it may have been pulled out of position. The remaining two participants voluntarily ended the trial early, both due to what they felt was a shorter catheter length. However, both had received standard length catheters, which were $400 \mathrm{~mm}$ in length. No catheters were removed over safety concerns.

\section{Secondary outcome: colonisation of original and trial catheters}

Twenty-nine of the 30 original and 28 of the 30 AUCs were collected. The three lost catheters fell out in the community and could not be retrieved. The original catheters and AUCs were matched ( $n=27$ pairs) and there were significantly fewer $(p=0.0088$, two-tailed $t-$ test) species of microorganisms attached to the AUC balloons compared to the matched control catheter. The pairs were well matched for the duration of catheterisation $(p=0.8428$, two-tailed t-test).

The lumens of two trial catheters were culture - negative and no lumens of original catheters were culture - negative. One possible concern at the start of the study was that eradication of organisms sensitive to the activity of the AUC would allow replacement by other organisms. However, this was not seen in the catheter analyses. For example, $E$. faecalis is not sensitive to the activity of the AUC yet there was no overgrowth of $E$. faecalis in the AUCs. In fact, five fewer AUC balloons and two fewer lumens contained $E$. faecalis compared to the control catheters. In general, the presence of all groups of organisms was reduced in the AUCs, with the exception of Pseudmonas spp. in which there were two more AUC lumens colonised with Pseudomonas spp. compared to the control catheters. The main limitation of this analysis is that the numbers are small and future studies will be needed to monitor the colonising microorganism populations. It is important to emphasise that, though reduction in CAUTI was mentioned by three participants in the free comments (Supporting Information Table 1), this trial was not designed to quantify efficacy.

\section{Discussion:}


221 Antimicrobial urinary catheters were produced and validated for use in this study,

222 which is the first human trial of this device. 84 AEs in 30 participants across 1681

223 catheterisation days were recorded, and of those only one was identified as being 'probably'

224 related to antimicrobial impregnation. This event was worse stinging than usual at

225 catheterisation and it resolved within 48 hours. The safety profile of the AUC appears

226 favourable. Patient acceptability was positive with $82 \%$ of participants rating the AUC as 'no

227 different' or better than their previous urinary catheters. Of the remaining $18 \%$ (5

228 participants) responding "a bit worse", two felt that their trial catheter was too short, despite it

229 being of identical length to their standard catheter; one changed the type of drainage device

230 and experienced disconnections; one experienced increased urinary urgency, and one

231 experienced pain on passing urine using a catheter valve.

232 Microbiological analysis of the participant's original and trial catheters demonstrated

233 a significant reduction of the number of species attached to the balloon of the AUCs.

234 Importantly, the use of the AUC did not increase the prevalence of MDR organisms or

235 increase the prevalence of microorganisms that are non-susceptible to the activity of the

236 AUC.

237 The CATHETER trial was a multi-centre randomised controlled trial of anti-septic

238 (silver-alloy and nitrofurazone coated) catheters for short-term use. Patients receiving

239 experimental catheters reported increased discomfort following catheterisation (silver

240 catheter $28.7 \%$, nitrofural catheter $38.9 \%$ ) and this was a motivation for our obtaining data

241 on safety and patient acceptability before undertaking larger studies. Our findings regarding

242 comfort and acceptability compared very favourably with these. Haematuria and septicaemia

243 were two recorded significant clinical events included in the CATHETER trial ${ }^{7}$. Haematuria

244 and blockage of the catheter due to a blood clot were recorded during this trial, but there

245 were alternative reasons for the presence of blood such as taking aspirin and the presence

246 of an enlarged prostate which is a recognised cause of haematuria ${ }^{8}$. During adjudication of

247 AE causality, as haematuria was not present without other predisposing factors and was no 
248 worse than previous episodes, the episodes were determined as 'possibly' related as it is

249 related to catheterisation but not necessarily catheterisation with the AUC.

250 The ESCALE trial, a trial of silver-alloy catheters in spinal cord injury patients, reported more AEs possibly related to catheterisation with the experimental catheters compared to standard catheters, including itching which was not reported in the control

253 group ${ }^{9}$. Other AEs captured by the ESCALE trial included haematuria, rash, blockage, and 254 suprapubic pain ${ }^{9}$. Rash related to catheterisation and suprapubic pain were not reported by 255 patients in our AUC safety trial. Blockage was reported during this trial.

Other unique AEs reported here included the sensation of needing to void, burning at the beginning and end of passing urine using a catheter valve, difficulty connecting the catheter bag to the catheter, and the catheter drainage system 'pushing off' the catheter connection. While these were mild events and mostly associated with the catheter drainage systems, they were still reported by participants as part of their catheter management. The base silicone urinary catheters that were impregnated with antimicrobials may have been from a different manufacturer than their normal catheter, which could have affected what the catheter user perceives as 'normal' for their catheter.

Although the follow-up was short, any AEs relating to the composition of the catheter material are likely to have manifested in the time period studied. Further studies will confirm long-term tolerability as well as clinical efficacy and will benefit from a control arm for comparison. Other limitations include that this was an unblinded study and this may have introduced an element of bias of the participant's in reporting. Although multi-centre trials provide a better generalisability of the results and therefore increased external validity, the participants represented many health conditions and were managed throughout several districts once catheterised with the trial catheter. Therefore, they were managed as standard according to their local policies and guidelines, which were not influenced by the clinical trial. In this trial participants were excluded if they not did have sensation in the urethra

274 and/or bladder as they would be unable to self-report some symptoms and also for their 275 safety. If the AUC were to cause irritation, allergy or discomfort both the participant and 
276 research team would be unaware. This by extension excluded patients with spinal cord injury

277 or cauda equina syndrome. Likewise, many patients with impaired cognitive capacity may

278 require a urinary catheter but were not eligible to participate due to the possible inability to

279 self-report new symptoms or adhere to the telephone interview schedule. These exclusion

280 criteria were put in place to protect patients and to preserve the accuracy of the data

281 collected. They will be included in a further randomised controlled trial of efficacy.

282

283 Conclusions:

284 The AUC has an advantageous safety profile and was an acceptable alternative

285 catheter to the majority of trial participants. Information gained from this trial will support

286 future regulatory applications for commercialisation and larger randomised controlled studies

287 of efficacy of the AUC.

288

289

290

References:

291

292 1. Loveday H, Wilson J, Pratt R, et al. epic3: National evidence-based guidelines for preventing healthcare-associated infections in NHS hospitals in England. $J$ Hosp

294 Infect. 2014:S1-S70.

295

296

297

298

2. Pickard R, Lam T, MacLennan G, et al. Antimicrobial catheters for reduction of symptomatic urinary tract infection in adults requiring short-term catheterisation in hospital: a multicentre randomised controlled trial. Lancet. 2012;380(9857):1927-

299

3. Fisher L, Hook A, Ashraf W, et al. Biomaterial modification of urinary catheters with antimicrobials to give long-term broadspectrum antibiofilm activity. J Control Release. 2015. 
302 4. Bayston R, Fisher L, Weber K. An antimicrobial modified silicone peritoneal catheter 303 with activity against both Gram-positive and Gram-negative bacteria. Biomaterials. $304 \quad 2009 ; 30(18): 3167-3173$.

305 5. Bayston R, Inventor; University of Nottingham, assignee. Medical devices and $306 \quad$ methods of making medical devices. 2007.

6. World Health Organization. The use of the WHO-UMC system for standardizes case causality assessment 2002 ; http://www.who.int/medicines/areas/quality safety/safety efficacy/WHOcausality ass

7. Pickard R, Lam T, Maclennan G, et al. Types of urethral catheter for reducing

8. Vasdev N, Kumar A, Veeratterapillay R, Thorpe AC. Hematuria secondary to benign prostatic hyperplasia: retrospective analysis of 166 men identified in a single one stop hematuria clinic. Curr Urol. 2013;6(3):146-149.

9. Bonfill X, Rigau D, Esteban-Fuertes M, et al. Efficacy and safety of urinary catheters with silver alloy coating in patients with spinal cord injury: a multicentric pragmatic randomized controlled trial. The ESCALE trial. Spine J. Nov 2017;17(11):1650-1657.

324 Table 1: Inclusion and exclusion criteria

Table 2: Participant demographics of consented participants 
327 Table 3: Description of adverse events (AEs), their relationship to antimicrobial impregnation

328 of trial catheters and their severity

329

330 Table 4: Replies from last recorded telephone interview of participants

331

332 Figure Legends:

333 Figure 1: STROBE flow diagram of participants involved throughout the trial pathway

334

335 
Response to Reviewers

Manuscript ID: NAU-18-0409

Reviewer 2:

3. Why is the data from the normal catheters included in table 3 ? It would be nice to compare the two for all these outcomes.

Answer: Table 3 presents the adverse events recorded during catheterisation with the antimicrobial-impregnated urinary catheter and not the normal catheters.

Reply: This is a rather unsatisfying answer. You have evaluated normal catheters for bacterial evaluation and compared in large sections the antimicrobial catheters with normal catheters (culturing etc.) and mirror tolerability between the two (being either patient reported or else). The primary aim of your study is safety and part of this is tolerability of this new catheter, but why is this then not systematically evaluated / reported by comparing them with the normal catheter, while you did do it for many other aspects of your study? Evaluation of the applicability of the new antimicrobial catheter will be based on an established balance between efficacy (reduced CAUTI and tolerability compared to normal catheters. Because there is no evaluation of efficacy of the catheter (amount of CAUTI'S) in this study, there should therefore have been be a systematic focus on tolerability by comparing table 3 parameters with a normal catheter in my opinion.

Reply to reviewer: Please find additional information in the manuscript (Lines 164-167) regarding efficacy data from baseline and with catheterisation with the antimicrobial catheter.

A comparison of tolerability between normal and antimicrobial catheters by patient self-reporting was carried out at each telephone interview. This information was summarised in Table 4.

According to the trial protocol, eligible participants were consented and after consent their normal catheter was removed and collected for analysis. The participant was catheterised with the antimicrobial catheter and followed up according to the telephone interview schedule. Therefore, normal catheters were not followed up in the same systematic manner as the aim of this study was to understand the safety profile of the AUC 'Specifically, this was determined by the rate of adverse events (AEs) attributable to the antimicrobials or the antimicrobial impregnation process' (lines 53-54). As we know which AEs are attributable to a normal urinary catheter, we determined that there was only one AE that was possibly related to antimicrobial impregnation, which was heightened stinging immediately following catheterisation. Comparison of patient responses when using the AUC with their experience of the previous, plain catheter (Table 4) showed that $\mathbf{4 2 . 3 1 \%}$ found it an improvement and $39.29 \%$ found it no different. In this way, we consider that we have done as the Reviewer requested, in that we have a comparison of the AUC with the previous plain catheter. To express this in terms of individual responses for each patient would consume a large amount of space but would not, in our view, add useful data. 
A safety and patient acceptability pilot study of a novel antimicrobial urinary catheter for long-term use

\section{Supporting Information}

Method 1:

Three $1.0 \mathrm{~cm}$ segments from five catheters were individually immersed in $2.0 \mathrm{~mL}$ chloroform to extract the drugs. This was repeated twice more and the extracts in chloroform were pooled together and the chloroform was removed under constant air flow. The extracts were re-solubilised in $100 \%$ methanol (HPLC grade, Fisher Scientific). Analysis was carried out by an Agilent 1100 HPLC machine with a variable wavelength UV detector (Agilent Technologies Inc.) connected to a Chemstation operating system. Chromatographic separations were performed using an Eclipse XDB-C8 (5 $\mu \mathrm{m}$, i.d. $4.6 \mathrm{~mm} \times 150 \mathrm{~mm})$ column (Agilent Technologies). The mobile phase was a mixture of $10 \%$ acetonitrile (HPLC grade, Fisher Scientific) and aqueous sodium dihydrogen phosphate (Sigma-Aldrich) adjusted to $15 \mathrm{mM}$ and $\mathrm{pH} 2.5$. The organic phase was $100 \%$ methanol. A gradient method was employed and maintained a flow rate of $1.0 \mathrm{~mL} / \mathrm{min}$ and an injection volume of $5.0 \mu \mathrm{L}$ with each run lasting eight minutes. The starting solvent concentration was $90 \%$ aqueous phase, $10 \%$ methanol increasing to $90 \%$ methanol after one minute and decreasing to $10 \%$ methanol at four minutes. Eluted drugs were first read at a wavelength of $254 \mathrm{~nm}$ to detect rifampicin and sparfloxacin, and then $279 \mathrm{~nm}$ to detect triclosan. The retention times were approximately 3.0, 3.6, and 4.5 minutes for sparfloxacin, rifampicin, and triclosan, respectively. 
2

3

4

5

6

7

8

9

Table 1: Thematic analysis of free text responses to the question 'How would you rate this catheter compared to your usual?' for the first and last telephone interviews along with the number of free responses fitting into each sub-theme

\begin{tabular}{|c|c|c|c|c|c|}
\hline \multicolumn{2}{|c|}{ First Interview } & \multirow[t]{2}{*}{ Number } & \multicolumn{2}{|c|}{ Last Interview } & \multirow[t]{2}{*}{ Number } \\
\hline Main Themes & Sub-Themes & & Main Themes & Sub-Themes & \\
\hline \multirow{2}{*}{$\begin{array}{l}\text { No noticeable } \\
\text { difference to } \\
\text { previous } \\
\text { catheters }\end{array}$} & $\begin{array}{l}\text { Too soon to } \\
\text { make a } \\
\text { judgement }\end{array}$ & 6 & $\begin{array}{l}\text { No noticeable } \\
\text { difference to } \\
\text { previous catheters }\end{array}$ & $\begin{array}{l}\text { Cannot tell a } \\
\text { difference }\end{array}$ & 9 \\
\hline & $\begin{array}{l}\text { Cannot tell a } \\
\text { difference }\end{array}$ & 8 & \multirow[t]{3}{*}{$\begin{array}{l}\text { Catheter } \\
\text { maintenance }\end{array}$} & Infection reduction & 3 \\
\hline \multirow[t]{2}{*}{$\begin{array}{l}\text { Catheter } \\
\text { maintenance }\end{array}$} & Urine flow & 4 & & $\begin{array}{l}\text { Leakage and } \\
\text { bypassing }\end{array}$ & 2 \\
\hline & $\begin{array}{l}\text { Leakage and } \\
\text { bypassing }\end{array}$ & 3 & & Catheter valve issues & 2 \\
\hline \multirow[t]{2}{*}{$\begin{array}{l}\text { Catheter } \\
\text { comfort }\end{array}$} & $\begin{array}{l}\text { Stinging and } \\
\text { soreness }\end{array}$ & 6 & \multirow[t]{3}{*}{ Catheter comfort } & Improved comfort & 6 \\
\hline & $\begin{array}{l}\text { Improved } \\
\text { sensation }\end{array}$ & 3 & & Discomfort & 3 \\
\hline \multirow[t]{4}{*}{ Mental Health } & \multirow{4}{*}{$\begin{array}{l}\text { Beneficial } \\
\text { impact on } \\
\text { mental health }\end{array}$} & \multirow[t]{4}{*}{2} & & Catheter material & 1 \\
\hline & & & Mental Health & $\begin{array}{l}\text { Beneficial impact on } \\
\text { mental health }\end{array}$ & 2 \\
\hline & & & \multirow{2}{*}{$\begin{array}{l}\text { Participant } \\
\text { satisfaction }\end{array}$} & $\begin{array}{l}\text { Desire to keep the } \\
\text { AUC }\end{array}$ & 3 \\
\hline & & & & $\begin{array}{l}\text { Desire to have a } \\
\text { second AUC }\end{array}$ & 2 \\
\hline
\end{tabular}


Table 1:

\begin{tabular}{|c|}
\hline Inclusion Criteria \\
\hline Age: 16 years old or greater \\
\hline $\begin{array}{l}\text { Currently fitted with a urinary catheter for at least } 28 \text { days and } \\
\text { will require another urinary catheter for } 28 \text { days or greater }\end{array}$ \\
\hline Able to understand written English and speak English fluently \\
\hline Able to verbally respond and to speak on the telephone \\
\hline Exclusion Criteria \\
\hline Pregnant or likely to become pregnant \\
\hline Adults lacking the ability to consent for themselves \\
\hline 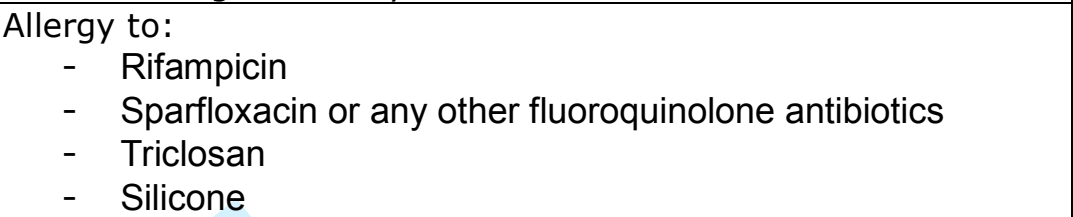 \\
\hline History of uncontrolled/unmanageable autonomic dysreflexia \\
\hline
\end{tabular}

Table 2:

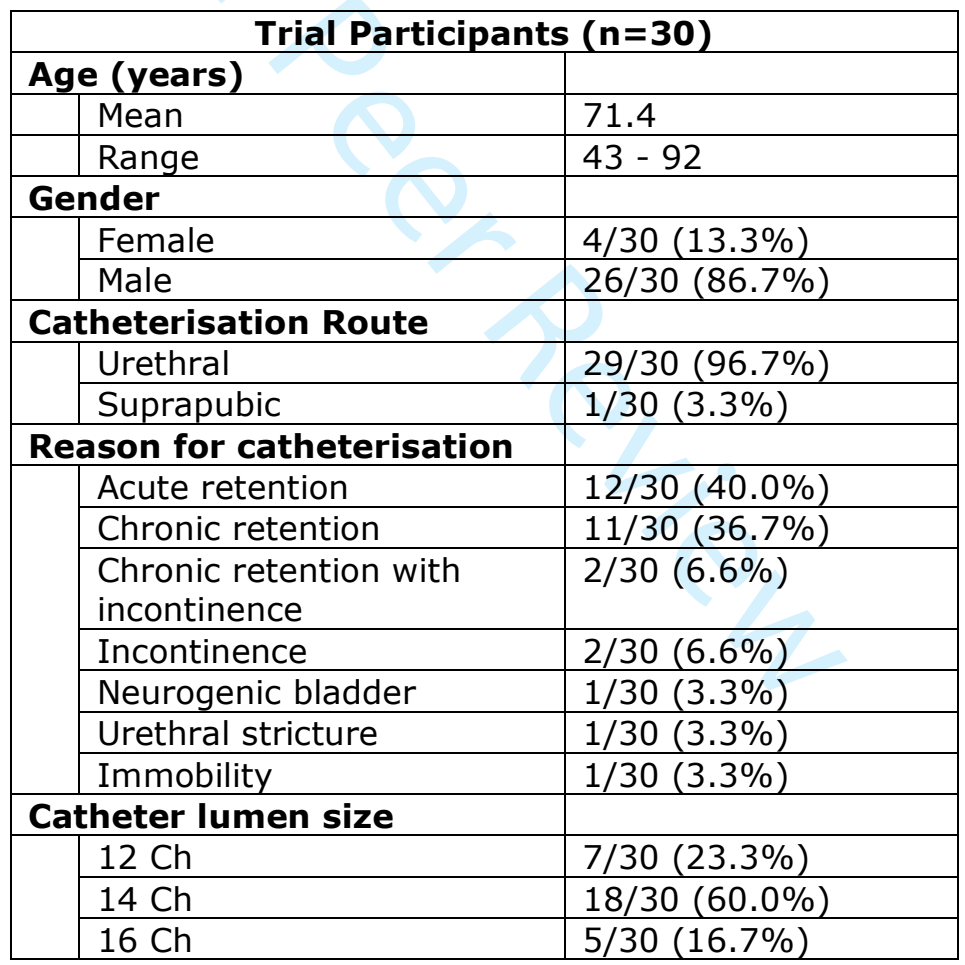


Table 3:

\begin{tabular}{|c|c|c|c|c|}
\hline \multirow{2}{*}{$\begin{array}{l}\text { AE relationship to antimicrobial } \\
\text { impregnation of the trial catheters }\end{array}$} & \multicolumn{4}{|c|}{ Number of AEs } \\
\hline & Mild & Moderate & Severe & Total \\
\hline AE 'Unrelated' & 37 & 14 & 5 & $\begin{array}{l}56 \\
(66.67 \%)\end{array}$ \\
\hline AE 'Unlikely' & 4 & 1 & $\mathbf{0}$ & $5(5.95 \%)$ \\
\hline Pelvic infection & 1 & & & 1 \\
\hline Dizzy after catheterisation & 1 & & & 1 \\
\hline Catheter fell out spontaneously & 1 & & & 1 \\
\hline Dizziness/generally unwell & & 1 & & 1 \\
\hline $\begin{array}{l}\text { Catheter displaced due to bag being full } \\
\text { and falling off catheter strap }\end{array}$ & 1 & & & 1 \\
\hline AE 'Possibly' & 19 & 3 & $\mathbf{0}$ & $\begin{array}{l}22 \\
(26.19 \%)\end{array}$ \\
\hline CAUTI & 2 & 2 & & 4 \\
\hline Blockage & 4 & 1 & & 5 \\
\hline $\begin{array}{l}\text { Burning at the beginning and end of } \\
\text { passing urine via the flip flow valve }\end{array}$ & 2 & & & 2 \\
\hline $\begin{array}{l}\text { Early catheter change due to perceived } \\
\text { shorter length }\end{array}$ & 2 & & & 2 \\
\hline Catheter expulsion & 1 & & & 1 \\
\hline Bypassing catheter & 1 & & & 1 \\
\hline Sensation of needing to void & 1 & & & 1 \\
\hline $\begin{array}{l}\text { Catheter bag/valve pushing off } \\
\text { connection }\end{array}$ & 1 & & & 1 \\
\hline Haematuria & 1 & & & 1 \\
\hline Stinging & 1 & & & 1 \\
\hline Testicular ache & 1 & $\bar{P}$ & & 1 \\
\hline $\begin{array}{l}\text { Difficulty connecting the catheter bag to } \\
\text { catheter }\end{array}$ & 1 & 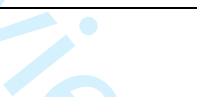 & & 1 \\
\hline Small sore on foreskin & 1 & $\sqrt{0}$ & & 1 \\
\hline AE 'Probably' & 1 & $\mathbf{0}$ & $\mathbf{0}$ & $1(1.19 \%)$ \\
\hline $\begin{array}{l}\text { Heightened stinging following } \\
\text { catheterisation }\end{array}$ & 1 & & & 1 \\
\hline
\end{tabular}

Table 4:

\begin{tabular}{|l|l|l|}
\hline Telephone interview question & \multicolumn{2}{|c|}{ Participant Responses } \\
\cline { 2 - 3 } & Response & $\begin{array}{l}\text { Percentage of } \\
\text { responses }\end{array}$ \\
\hline \multirow{2}{*}{$\begin{array}{l}\text { 'How would you rate this catheter } \\
\text { compared to your usual catheter?' }\end{array}$} & Much better & $35.17 \%$ \\
\cline { 2 - 3 } & A bit better & $7.14 \%$ \\
\cline { 2 - 3 } & No different & $39.29 \%$ \\
\cline { 2 - 3 } & A bit worse & $17.86 \%$ \\
\cline { 2 - 3 } & Much worse & $0.0 \%$ \\
\hline \multirow{2}{*}{$\begin{array}{l}\text { 'Have you had any pain from the } \\
\text { catheter' }\end{array}$} & Less than usual & $32.14 \%$ \\
\cline { 2 - 3 } & About the same & $57.14 \%$ \\
\cline { 2 - 3 } & More than usual & $10.71 \%$ \\
\hline
\end{tabular}


396 potentially eligible patients identified from GP and DN records

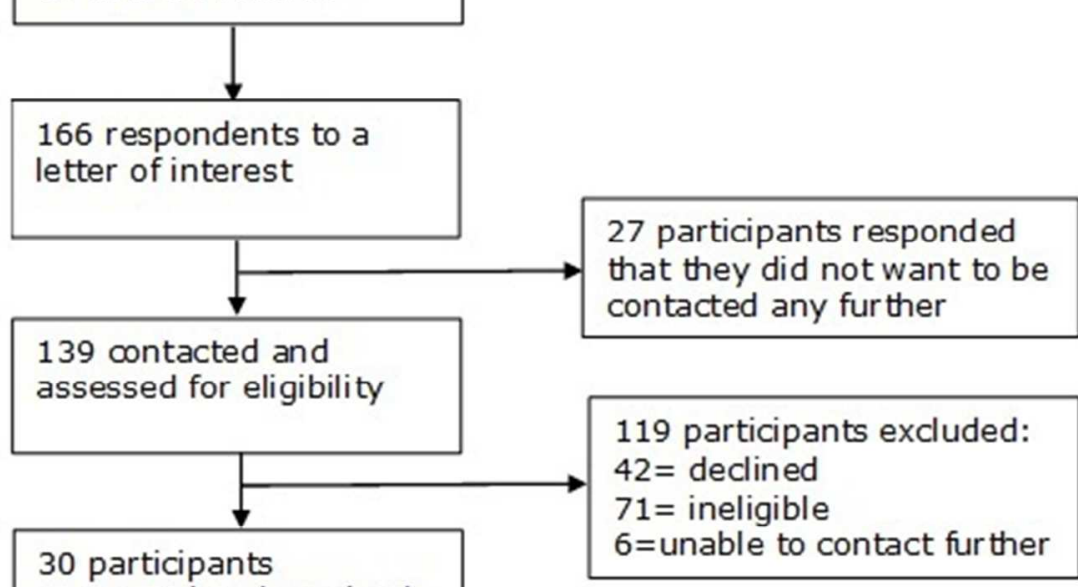

consented and received

the antimiarobial urinary catheter

30 participants induded in the safety analysis 